\title{
„This is a process not a protest“ Globale urbane Proteste, Betriebsbesetzungen zur Produktion unter Arbeiterkontrolle und lokale Selbstverwaltung
}

\author{
Dario Azzellini
}

\begin{abstract}
Der Beitrag verknüpft die Platzbesetzungen seit 2011 mit den Betrieben unter Arbeiterkontrolle, die ab 2000 in Lateinamerika und darüber hinaus entstanden, sowie mit Formen kollektiver lokaler Selbstverwaltung. Diesen Praxen sind bei allen Unterschieden grundlegende Charakteristika gemeinsam. Sie konstruieren Räume der Produktion sozialer Alternativen. Ich arbeite die Gemeinsamkeiten heraus und argumentiere, dass sie Teil globaler, nicht-staatszentrierter Transformationsperspektiven sind. Sie machen eine wachsende Tendenz sichtbar, die sich an direkter Demokratie, Selbst-Organisierung und Autonomie orientiert. Entgegen der Kritik der Strategielosigkeit wird gezeigt, dass die Strategie darin besteht, in den selbst produzierten Räumen Elemente der angestrebten Veränderungen zu entwickeln und zu erproben. Die Beteiligten sehen die Praktiken nicht als Nische, sondern als gesamtgesellschaftliche Alternative. Die Praktiken werden via Netzwerke verknüpft und bilden neue räumliche Konfigurationen.
\end{abstract}

Ersteinreichung: 18. April 2016; Veröffentlichung online: 29. November 2016

An english abstract can be found at the end of the document.

\section{Einleitung}

Die Verbindungen und Gemeinsamkeiten zwischen den verschiedenen Protestbewegungen seit Beginn der aktuellen Krise 2008 von Tunesien und Island über die weltweiten Platzbesetzungen bis zu den aktuellen französischen Protesten wurden von zahlreichen Autor_innen herausgestellt (Arenas 2014, Castañeda 2012, Castells 2012, Cossar-Gilbert 2016, Mason 2013, Roos/ Oikonomakis 2014, Sitrin/Azzellini 2014). Die Bewegungen entstehen in einer Krise der Repräsentation und teilen die Kritik - ob in autoritären Regimen oder repräsentativen Demokratien - an der Repräsentation. Sie empfinden diese als undemokratisch und praktizieren Formen direkter Demokratie (Arenas 2014, Castañeda 2012, Juris/Ronayne/Shokooh-Valle/ Wengronowitz 2012, Ressler 2012, Sitrin/Azzellini 2014, Yörük 2014).

Selten und eher punktuell als systematisch sind hingegen die Gemeinsamkeiten der neuen globalen Bewegungen, und deren Orientierungen auf direkte Demokratie, Kollektivität, Solidarität, Selbstverwaltung und Autonomie, mit zwei weiteren Bewegungen der vergangenen Jahre untersucht worden: (1) Den rückeroberten Betrieben unter Arbeiterkontrolle (RBA). Gemeint sind Hunderte von Betriebe, die seit der Jahrtausendwende 
vornehmlich in Lateinamerika von Arbeiter_innen besetzt wurden, um selbstverwaltet zu produzieren. (2) Den diversen Systemen lokaler Selbstverwaltung von Mexiko über Venezuela bis Kurdistan, die hier Kommunen genannt werden. Einige Autor_innen führen die neuen globalen Bewegungen und einzelne Beispiele von RBA oder Kommunen als miteinander verwandte Bestandteile einer Produktion und Erprobung gesellschaftlicher Alternativen an, die nicht in der Tradition der seit 1848 dominanten Form der repräsentierenden Partei oder Gewerkschaft steht (Sitrin/Azzellini 2014: 14-39, Hardt/ Negri 2009; 2013, Harvey 2012, Holloway 2010, Ross 2015, Teivainen 2016).

Die alternativen politischen Praxen der neuen globalen Bewegungen werden von Teivainen (2016) als „transnationaler libertärer Sozialismus“ bezeichnet. Lopes (2014) analysiert die neue Orientierung in urbanen Bewegungen und nennt das Phänomen „libertäre Wende“. In meinem Beitrag zeige ich auf, auf welche Weise die Gemeinsamkeiten in der alternativen Raumproduktion neue globale Bewegungen, RBA und Kommunen verbinden. Damit sollen diese nicht miteinander gleichgesetzt werden. Sie unterscheiden sich voneinander und untereinander in Dauer, Form, Umfang und Reichweite. Darauf wirken zahlreiche Faktoren ein. Ortsspezifische Dynamiken prägen alle gesellschaftlichen Kämpfe (Harvey 1995). Es soll auch keine vermeintlich gemeinsame Front aus den verschiedenen Praktiken und Kämpfen konstruiert werden. Vielmehr geht es darum herauszustellen, wie in ihnen eine politische Kultur sichtbar wird, die eine Alternative darstellt zu der traditionellen Organisierung von Protest im Bezugsrahmen der Repräsentation sowie des Staates und seiner Institutionen.

Der Zusammenbruch des Realsozialismus und die neoliberale Globalisierung haben Ansätze befördert, die eher in der historischen Tradition der Pariser Kommune stehen als in der des autoritären und staatszentrierten Sozialismus. Die Renaissance rätebasierter Selbstorganisierung erfasst Praxis und Debatten. Zeitgenössische Theoriekonzepte, die in Rätemodellen und Selbstverwaltung gründen, erfahren verstärkt Beachtung. Dazu gehören etwa die kommunalen Konsum- und Produktionskreisläufe des in Lateinamerika stark rezipierten István Mészáros (1995), der „demokratische Föderalismus“ von Murray Bookchin (Biehl/Bookchin 1997, Bookchin 1992; 2015), der vom kurdischen Befreiungskampf übernommen wurde (Flach/Ayboğa/ Knapp 2015) und das Konzept „Parecon“von Michael Albert (2006). Diverse Diskussionen um Alternativen zum kapitalistischen System, die im Kontext der Krise erfolgten, verwiesen ebenfalls auf demokratische Rätemodelle (Bonnet 2014, Esteva 2009, Demirovic 2009, Roth 2008, Wolff 2012, Zelik/ Tauss 2013).

Tahrir, 15-M in Spanien, Occupy in den USA, Occupy in London, Griechenland, Kanada, Brasilien und die Gezi-Park-Bewegung - die Platzbesetzungen breiteten sich weltweit aus (Harvey 2012: 161, Mason 2013, Roos/Oikonomakis 2014, Sitrin/Azzellini 2014).[1] Sie sind „eine sehr lokale und spezifische Strategie und eine transnationale oder globale Praxis zugleich“ (Feigenbaum/Frenzel/McCurdy 2013: 2, Übers. d. A.). Es wird ein zentraler Platz besetzt und in einen Ort verwandelt, um darüber zu diskutieren was in der Gesellschaft falsch läuft und was dagegen zu tun ist. Die Platzbesetzungen drücken ein Bedürfnis nach einem Raum aus, in dem ein freier Austausch möglich ist und die Regeln dafür gemeinsam festgelegt 
werden. Es entsteht ein „politischer Commons“ (Harvey 2012: 161). Auf den besetzten Plätzen wird versucht „die soziale Reproduktion und Neuschaffung des täglichen Lebens in Weisen zu ermöglichen, die den Status Quo anfechten“(Feigenbaum/Frenzel/McCurdy 2013: 10; Übers. d. A.). Im Unterschied zu RBA und Kommunen geschieht dies auf den Plätzen aber nur temporär.

Anders als üblicherweise bei Protestcamps seit den 1960ern geht es bei den Platzbesetzungen ab 2011 nicht um den Protest gegen eine Maßnahme oder einen Akteur (also „contentious politics“, McAdam/Tarrow/Tilly 2001), sondern um eine systemische Unzufriedenheit, die in die Öffnung von Räumen zur Erprobung von Alternativen mündet. So lautete eine weit verbreitete Losung von Occupy LSX (London) bis Occupy US: „Dies ist kein Protest, sondern ein Prozess". Ganz gleich, was nun der konkrete Anlass für die Platzbesetzungen war, immer ging es dabei um wesentlich mehr. Stand bei Occupy die Macht der Konzerne und Gier der Eliten im Vordergrund, waren unter anderem auch Themen wie Zwangsräumungen, Ökologie, direkte Demokratie, Studienschulden, die Militarisierung der Polizei, Gender und Rassismus stark präsent (Arenas 2014: 434). Ganz ähnlich war es auch auf den anderen Plätzen weltweit. Sie alle hatten gemeinsam, dass sie eine gesamtgesellschaftliche Mobilisierung und Veränderung anstrebten (Byrne 2012, Castañeda 2012, Graeber 2012, Interview Lozada (2012):, Ressler 2012, Roos/Oikonomakis 2014, Schumacher/Osman 2012, Sitrin/Azzellini 2014, Yörük 2014).

Entgegen oberflächlicher Betrachtung und weit verbreiteter Ansicht ist das, was auf den Plätzen wirkte und sichtbar wurde, nicht verschwunden. Allen Platzbesetzungen gingen zahlreiche soziale Kämpfe voraus, in denen spätere Praktiken und Inhalte angelegt waren (Ali 2012, Castañeda 2012, Roos/ Oikonomakis 2014: 125-128, Schumacher/Osman 2012, Sitrin/Azzellini 2014; Sowers/Toensing 2012, Yörük 2014). Die meisten Platzbesetzungen beschlossen nach einiger Zeit selbst ihre Auflösung, um an anderen Orten aktiv zu werden, einige wurden auch gewaltsam geräumt. Es erfolgte eine bewusste Reterritorialisierung, die in bestimmten Stadtteilen und konkreten Projekten vollzogen wurde. In Spanien folgten Bewegungen bezüglich Bildung, Gesundheitsversorgung und Diskriminierung gegen Frauen, die auf Versammlungen und direkter Demokratie beruhten. Dort, in Griechenland sowie in den USA entstanden zahlreiche Kooperativen und Netzwerke von Produzent_innen und Verbraucher_innen. Strike Debt, die US-Bewegung gegen Privatverschuldung durch Studium oder Gesundheitsversorgung, ist aus Occupy hervorgegangen; die Bewegung gegen Zwangsräumungen in den USA und Spanien wurden massiv gestärkt (Roos/Oikonomakis 2014, Sitrin/Azzellini 2014). Tahrir führte zu einem enormen Zuwachs an betrieblicher Selbstorganisation und unabhängigen Gewerkschaften (Alexander/ Bassiouny 2014: 224-251); zudem entstanden mindestens zwei RBA (Azzellini 2015b), bis ein Militärputsch eine massive Repression entfachte.

Besetzungen von Betrieben mit dem Ziel, unter Selbstverwaltung zu produzieren, hat es historisch vereinzelt in verschiedenen Ländern gegeben. Zu einer breiteren Praxis wurden sie mit der Krise 2000/2001, als Arbeiter_innen in Argentinien damit begannen, ihre schließenden Betriebe zu besetzen. Die Besetzungen weiteten sich auch auf Uruguay, Brasilien und Venezuela aus. 2015 existierten in Argentinien etwa 360 RBA, in Brasilien 70, 
in Uruguay 22 und in Venezuela etwa 80 (Azzellini 2014, 2015c, 2016, Chedid et al. 2013, Sardá de Faria/Novaes 2012, Rieiro 2015). Vereinzelte RBA entstanden auch in anderen lateinamerikanischen Ländern und Asien (Azzellini 2015a; Azzellini/Ness 2012). Im Zuge der aktuellen Krise kamen in Argentinien fast 60 und in Venezuela etwa zwei Dutzend neue RBA hinzu. Auch in Italien, Frankreich, Griechenland, Bosnien, Kroatien, Tunesien sowie in den USA und in der Türkei entstanden einzelne RBA (Azzellini 2015b, 2015c). RBA sind in allen industriellen Sektoren entstanden (Metall, Textilien, Lebensmittel, Chemie und Baustoffe) sowie zunehmend im Dienstleistungsbereich (Gaststätten, Kliniken, Hotels und Transport) und im Bereich Medien (Azzellini 2015a; Azzellini/Ness 2012).

Eine Reihe von Eigenschaften unterscheiden RBA von anderen selbstverwalteten Betrieben und von traditionellen Kooperativen. Die RBA bilden eine offensive Strategie in einer defensiven Situation. Anstatt zu resignieren nehmen die Arbeiter_innen ihr Schicksal selbst in die Hand und zeigen eine Lösung auf. Ein zuvor hierarchisch-kapitalistisch organisierter Betrieb mit dem Primärziel, den Mehrwert zu steigern und abzuschöpfen, wird in einen demokratisch selbstverwalteten Betrieb verwandelt, in dem das Wohlergehen der Arbeiter_innen im Mittelpunkt steht. Ein RBA ist ein sozialer und ökonomischer Prozess. Es entstehen neuartige soziale Beziehungen und es verändert sich die Arbeitsweise. Das Privateigentum an Produktionsmitteln wird in kollektives soziales Eigentum umgewandelt. Die Form, in der dies geschieht, stellt das Privateigentum an Produktionsmitteln infrage. Die Produktion unter demokratischer Selbstverwaltung als Antwort auf den Angriff des Kapitals reaffirmiert die Identität der Arbeiter_innen als Arbeiter_innen, allerdings ohne Chef, während zugleich gezeigt wird, dass eine andere Art zu arbeiten möglich ist (CDER 2014, Chedid et al. 2013: 27, 30, Ruggeri 2014: 16, Sitrin 2012).

Lokale Selbstverwaltung mittels direkter Demokratie als linke, sozialistische Perspektive im größeren Rahmen tauchte nach dem Fall des Realsozialismus erstmals in indigenem Kontext mit dem Kampf der Zapatistas in Chiapas, Mexiko, auf. Seitdem sind in verschiedenen Weltregionen ähnliche Ansätze der politischen Organisierung sozialistischer Gesellschaften als Föderationen von Kommunen zu finden. Ich beziehe mich hier vorwiegend auf Chiapas und Venezuela, sowie in geringerem Maße auf Guerrero, Oaxaca und Kurdistan.

Die neuen Praktiken lokaler Selbstverwaltung in Lateinamerika sind stark von indigenen Widerstandserfahrungen und Vorstellungswelten geprägt. Diese haben auf der einen Seite viel gemeinsam mit der sozialistischen Kommunentradition, die vor dem Aufkommen des Staatssozialismus als Vorstellung von Sozialismus hegemonial war, und andererseits mit dissidenten sozialistischen Strömungen wie Rätekommunismus, libertärem Sozialismus und Anarchosyndikalismus. Gustavo Esteva aus Oaxaca unterstreicht den „kommunitären Impetus“ dem der Sozialismus entstammt. „Die Communitys erscheinen als eine Alternative, weil in ihnen die Einheit zwischen Politik und Ort wieder hergestellt wird und das Pueblo eine Form hat, in der es seine Macht ausüben kann, ohne sich dem Staat ergeben zu müssen" (Esteva 2009, Übers. d. A.). 
In Kurdistan knüpft die Selbstverwaltung sowohl an lokale kommunitäre Traditionen wie auch an sozialistische Konzepte an. Die zuvor marxistisch-leninistische nationale Befreiungsbewegung PKK orientierte sich ab Ende der 1990er am demokratischen Konföderalismus von Bookchin. 2005 wurden die PKK und die zahlreichen Organisationen um sie herum reorganisiert als KCK, Assoziation der Communitys Kurdistans (Jongerden/ Hamdi 2013). Es folgte der Aufbau von Rätestrukturen in Nordkurdistan (Türkisch-Kurdistan), die bis heute klandestin arbeiten. 2007 weiteten sich die klandestinen Rätestrukturen auf Westkurdistan (Nordsyrien) aus und wurden nach dem Kollaps der Regierungskontrolle ab 2011 öffentlich (Flach/ Ayboğa/Knapp 2015, Jongerden/Hamdi 2013: 173).

Es folgen nun einige Aspekte der sozial-utopischen Antizipation, gemäß der englischsprachigen Debatte „Präfiguration“ genannt, in den Platzbesetzungen, RBA und Erfahrungen lokaler Selbstverwaltung. Dabei werden bereits Gemeinsamkeiten in der Produktion gesellschaftlicher Alternativen in den von ihnen produzierten Räumen herausgestellt. Im Anschluss erörtere ich die Frage der Autonomie, die direktdemokratische Entscheidungsfindung und die Herstellung räumlicher Konfigurationen mittels Netzwerke als zentrale Elemente der Raumkonstruktion. Daraus wird deutlich, wie diese Praktiken in einem gemeinsamen Rahmen gesellschaftlicher Transformationsstrategien eingeordnet werden können.

Angesichts der Kritik, vor allem von Geograph_innen, allgemeine Interpretationen sozialer Kämpfe litten häufig an mangelnden empirischen Grundlagen (Arenas 2014: 434), beziehe ich mich zentral auf Aussagen, Praktiken und Vorstellungen der direkt Beteiligten. Auch die verwendete akademische Literatur stammt meist von Autor_innen, die an den Praktiken beteiligt waren oder sind.[2]

\section{Sozialutopische Antizipation statt Repräsentation}

Neue globale Bewegungen, RBA und Kommunen produzieren Räume der sozialutopischen Antizipation. Es sind Räume, wie sie ganz ähnlich von Ernst Bloch als „konkrete Utopie“ (1973), von Walter Benjamin als „Jetztzeit“(1965), von David Harvey als „Räume der Hoffnung“ (2000) und von John Holloway als „Risse im Kapitalismus“ (2010) beschrieben werden. Da diese Praxis charakteristisch ist für viele der neuen Bewegungen, werden sie auch als präfigurative Bewegungen bezeichnet (Maeckelbergh 2011, Sitrin 2006; 2013, Teivainen 2016, van de Sande 2013). Teivainen (2016) schlägt vor, von „demokratischer Präfiguration “ zu sprechen, da historisch auch religiöse, esoterische, völkische und faschistische präfigurative Bewegungen existiert haben.

Präfigurative Bewegungen erzeugen ,in ihren aktuellen sozialen Beziehungen die Zukunft" (Sitrin 2006: 4; Übers. d. A.). Die Mittel werden nicht dem Ziel untergeordnet. Es geht nicht um die Implementierung eines Programms, sondern um einen Prozess mit unklarem Ausgang und einer ,inhärent experimentellen und empirischen Praxis" (van de Sande 2013: 232, Übers. d. A.). Der Zapatista-Slogan „Fragend gehen wir voran“ drückt dieses Verhältnis aus.

Auf den besetzten Plätzen entstanden überall thematische Arbeitsgruppen, in denen diskutiert und politische Aktionen vorbereitet wurden, und kollektive 
Strukturen, um die zur Erhaltung des Camps notwendigen Arbeiten zu organisieren. Dazu gehörten zu Beginn Infrastruktur, Verpflegung, Sicherheit sowie juristischer Beistand. Die größeren Platzbesetzungen verwandelten sich in kleine Städte, es entstanden Bibliotheken, Kinderbetreuung, Übersetzungsdienste und Schulen, es wurden Zeitungen, Webseiten, Kunst und Kultur produziert und politische Proteste, Aktionen und Veranstaltungen organisiert. Die Angebote waren in der Regel alle kostenlos, es galt, dass Partizipation nicht an mangelnden finanziellen Mitteln scheitern dürfe. Die Räume waren „Commons insofern die internen Beziehungen nicht die des Warenaustauschs waren, ein anti-hierarchischer Ethos oder ,Horizontalität überwogen, und grundlegende menschliche Bedürfnisse wie Sicherheit, Essen, Abfallbeseitigung, Gesundheit, Wissen und Unterhaltung selbstorganisiert waren" (Linebaugh 2014: 24). Alle Platzbesetzungen waren nach auBen hin offen und verfügten über Strukturen, um Interessierte zu empfangen. Einige der Platzbesetzungen waren aber auch mit rassistischen Vorfällen, sexuellen Übergriffen, Störer_innen, psychisch Kranken, Drogenkonsum und Odachlosen konfrontiert und mussten Weisen entwickeln, wie damit umgegangen werden konnte (Arenas 2014, Byrne 2012, Graeber 2012, Interview Lozada (2012), Juris/Ronayne/Shokooh-Valle/Wengronowitz 2012, Ressler 2012, Roos/Oikonomakis 2014, Sitrin/Azzellini 2014, Yörük 2014).

RBA sind dauerhafter angelegt als Platzbesetzungen und es besteht für die Beteiligten weniger die Möglichkeit, bei Unzufriedenheit einfach wegzugehen. Die direkte kollektive Verwaltung steht hier im Zentrum und es wird Zeit und Kraft darein investiert, eine andere Form der Arbeit zu praktizieren. RBA halten wesentlich häufiger Versammlungen ab als traditionelle Kooperativen (CDER 2014: 46). 88 Prozent der RBA in Argentinien führen regelmäßige Vollversammlungen durch, 44 Prozent davon wöchentlich (PFA 2010: 47). In Brasilien finden Vollversammlungen seltener statt. Doch zugleich finden, wie auch in anderen RBA, regelmäßige Koordinationssitzungen statt; 75 Prozent der brasilianischen RBA verfügen zudem über Wandzeitungen, 43 Prozent über regelmäßige Abteilungsversammlungen und elf Prozent über interne Publikationen (Chedid et al. 2013: 124). Ganz grundsätzlich gibt es in RBA keine Geheimisse und keine Repression, das heißt, es kann immer und überall über die RBA geredet werden - was auch geschieht.

Das Prinzip der Gleichheit betrifft in den meisten RBA auch die Bezahlung. Eine Untersuchung von 81 RBA in Argentinien ergab, dass 45 davon alle Beschäftigten gleich bezahlen, während bei weiteren 34 der Unterschied zwischen dem höchsten und niedrigsten Einkommen höchstens 33 Prozent beträgt (PFA 2010: 55-56). Die RBA in den USA, Italien, Frankreich, Griechenland und der Türkei zahlen ebenfalls gleiche Einkommen (Azzellini 2015b). Das gilt auch für zehn RBA in Venezuela und zwei in Uruguay, die ich besucht habe. Nur in Brasilien liegen große Einkommensunterschiede vor. In 15 von 50 untersuchten RBA war das höchste Einkommen sogar fünf bis zehn Mal so hoch wie das niedrigste (Chedid et al. 2013: 128). Da dies kollektiv beschlossen wurde, wäre weitere Forschung zu den Ursachen interessant.

Die Kommunen stellen zweifelsohne die weitestgehende und komplexeste Form der Präfiguration dar. Der Aufstand der Zapatistas führte 1994 als erstes zur Gründung von 32 „Rebellischen Autonomen Zapatistischen Landkreisen“ (MAREZ), bestehend aus einer unterschiedlichen Anzahl 
Autonomer Gemeinden. Sie funktionieren mit Rätesystem und direkter Demokratie und koordinieren sich auf höheren Ebenen, das heißt zwischen den autonomen Gemeinden besteht eine Koordination innerhalb der autonomen Landkreise, während diese wiederum auch miteinander koordiniert werden (Vergara-Camus 2014). Die Zapatistas widmeten sich zunächst dem Aufbau von Bildungs- und Gesundheitsversorgung sowie der Koordination der Landwirtschaft und dem Austausch von Agrarprodukten untereinander. Parallel dazu entwickelten sie ein alternatives Justizsystem und widmeten sie sich dem Aufbau von Kooperativen. Die Zapatistas reklamieren ,Autonomie‘. Neben indigenen Einflüssen ist der Zapatismus von mexikanischen sozialrevolutionären Strömungen, autonomem Marxismus und Anarchismus beeinflusst (EZLN 2016, Ramírez 2003, Vergara-Camus 2014).

Das Beispiel der Zapatistas führte zur Ausrufung selbstverwalteter indigener Gemeinden in Oaxaca und Guerrero. Im Bundesstaat Oaxaca mit mehrheitlich indigener Bevölkerung gibt es eine starke Selbstverwaltung in ländlichen Gemeinden. In der gleichnamigen Hauptstadt Oaxaca verwandelte sich das Protestcamp einer Lehrergewerkschaft nach der brutalen Räumung durch die Polizei am 14. Juni 2006 in einen allgemeinen Aufstand. Polizei und Stadtverwaltung verließen die 600.0oo Einwohner_innen zählende Stadt, die von Tausenden von Barrikaden geschützt wurde. Bis zur Erstürmung durch Sondereinheiten der Polizei Ende Oktober wurden Alltag und politische Aktionen von der Bevölkerung selbst und mittels der neu gegründeten Popularen Versammlung der Völker Oaxacas (APPO) organisiert. Die APPO war eine Koordination von Sprecher_innen von über 300 politischen und sozialen Organisationen und Gruppen von Landarbeiter_innen, Studierenden, indigenen Gruppe und Nachbarschaften. Der indigene Erfahrungsschatz stellte ein bedeutendes Element in der „Kommune von Oaxaca“ dar. Zugleich aber ereignete sich der Aufstand in der Stadt, wo die Bevölkerung mehrheitlich nicht indigen ist (Arenas 2014, Beas Torres 2007, Esteva 2009; 2010).

In Venezuela entstand die lokale Selbstverwaltung ab 2004 von unten durch linke Basisorganisationen in urbanen und ländlichen Regionen. 2005 griff die Regierung, auf Initiative von Präsident Hugo Chávez, den Ansatz der Kommunalen Räte auf. Diese bestehen in den Städten aus 150 bis 400 Wohneinheiten und in ländlichen Regionen aus mindestens 20. Ihre Struktur existiert parallel zu den Institutionen der repräsentativen Demokratie und ist diesen nicht unterworfen. Die Räte arbeiten als direktdemokratische Nachbarschaftsversammlungen mit verschiedenen thematischen Komitees, in denen Projekte für die Community erarbeitet werden. Sie widmen sich Infrastrukturprojekten, Gesundheitsversorgung, Bildung, Sport, Kultur, Medien und zunehmend dem Aufbau kollektiv betriebener produktiver Projekte (Azzellini 2010: 261-299).

Ab 2007 entstanden comunas als Zusammenschlüsse von Kommunalen Räten und anderer im Territorium präsenter Basisorganisationen. Die Entscheidungen werden weiterhin in den Kommunalen Räten getroffen, die ihre Sprecher_innen entsenden. Eine gesetzliche Regelung für die comunas folgte 2012 (Azzellini 2013). Im März 2016 existierten in Venezuela 45.749 Kommunale Räte und 1.500 comunas.[3] Diese erhalten jährlich bis zu sechs Milliarden US-Dollar an staatlicher Finanzierung. Der Mechanismus 
der Bildung von comunas ist flexibel gestaltet. Die Beteiligten definieren selbst, welche gemeinsamen Projekte sie entwickeln und welche Aufgaben sie übernehmen. Das erleichtert den Organisierungsprozess und ermöglicht ein organisches Wachstum, doch ist die starke Überlagerung der kommunalen selbstorganisierten Räume mit dem staatlich-administrativen Raum Ursache häufiger Konflikte (Azzellini 2013; 2014). Die Räte klagen über Kooptationsversuche aus Institutionen und Politik, langwierige und intransparente bürokratische Verfahren in der Interaktion mit Institutionen sowie über Korruption und ein fehlerhaftes Management innerhalb der staatlichen Institutionen (Azzellini 2010: 310-312; 345-349, Sitrin/ Azzellini 2014: 240-244).

Der kommunale Sozialismus im Aufbau nährt sich aus kommunistischen und anarchistischen Einflüssen ebenso wie aus popularen, indigenen und afro-amerikanischen Erfahrungen (Azzellini 2013). So nennen die afro-venezolanischen Communitys in der Region Barlovento ihre comunas „cumbes“, wie die einst selbstverwalteten freien Siedlungen von Ex-Sklav_ innen in der Region. Die Schaffung von kommunalen Produktions- und Konsumkreisläufen, kombiniert mit Rätestrukturen verschiedener Art auf unterschiedlichen Ebenen, als Transition zum Sozialismus, geht stark auf István Mészáros (1995) zurück und ist von der kritischen Geografie beeinflusst, vor allem von Doreen Masseys Arbeit zu ungleichen und antidemokratischen Machtgeometrien (2009).

Die präfigurative Praxis der neuen globalen Bewegungen wird häufig als Strategielosigkeit kritisiert (Dean 2013, Demirovic 2014, Porcaro 2013, Roberts 2012, Wainwright 2012). Dabei wird davon ausgegangen, Strategie definiere klare Wege und Ziele und bedürfe einer vertikalen Organisierung, während Präfiguration kulturell und unorganisiert sei (Maeckelbergh 2011: 4-7). Letzteres lässt sich empirisch nicht bestätigen. Die präfigurativen Praktiken der neuen globalen Bewegungen, RBA und Kommunen zeichnen sich dadurch aus, dass es sich nicht um einen Rückzug handelt, um nach eigenen Vorstellungen zu leben, wie in einigen präfigurativen Bewegungen seit den 1960ern. Im Gegenteil - die Bewegungen machen Kapital und Staat, Orte und Räume streitig und eignen sie sich an. Luca Federici vom RBA RiMaflow in Mailand nennt das „konfliktive Selbstverwaltung“ (Azzellini/Ressler 2014). „Präfigurative Strategie“, so Maeckelbergh, „beinhaltet zwei grundlegende Praxen: Die der Konfrontation mit den existierenden politischen Strukturen und Entwicklung von Alternativen - keine von beiden könnte ohne die andere die erwünschten strukturellen Veränderungen erzielen“ (2011: 15, Übers. d. A.).

\section{Gemeinsame Aspekte der Raumproduktion}

Das Streben nach Autonomie und direkter Demokratie gehört zu den zentralen Gemeinsamkeiten in der Raumproduktion von Platzbesetzungen, RBA und Kommunen. Um etwas Neues produzieren zu können und nicht kooptiert zu werden oder vorhandene Formen zu reproduzieren, werden die Räume möglichst freigehalten von Einflüssen durch Staat und Kapital. Die Entscheidungsfindung mittels partizipativer und direkter Demokratie trägt entscheidend dazu bei, einen Raum für alle zu schaffen, den auch 
alle gleichberechtigt mitgestalten (Sitrin/Azzellini 2014: 134; 158-163). Die demokratische Entscheidungsfindung ist dabei gleichermaßen Weg und Ziel (Maeckelbergh 2011: 6-11, Polletta 2002: 199, Teivainen 2016: 25). Ich werde aufzeigen, wie durch Inspirationen, Kontakte und Netzwerke eine kulturelle und räumliche Konfiguration erfolgt, welche die verschiedenen Praktiken zusammenbringt und eine Strategie hin zu einer neuen Art der politischen Organisierung erkennen lässt, deren Prinzipien und Ziele sich in den Praktiken wiederspiegeln.

\section{Ein Raum Jenseits des Staates}

Der von Plätzen, RBA und Kommunen produzierte Raum ist wesentlich ein Raum jenseits des Staates und seiner Organisationsformen. Angesichts der Machtasymmetrie zu Gunsten der konstituierten Macht kann nur so etwas Neues entstehen ohne dem Druck zu erliegen, die von Staat und Kapital vorgegebenen Formen zu übernehmen. Plätzen, RBA und Kommunen ist daher ein Streben nach Autonomie gemeinsam. Autonomie meint Prioritäten selbst zu bestimmen und Entscheidungen ohne den Druck anderer Akteure zu treffen. Das eigene Projekt steht im Mittelpunkt, nicht Staat oder Kapital. Die Ausgangsbasis sind die eigenen Wünsche und Bedürfnisse, nicht der Rahmen, der von anderen Akteuren vorgegeben wird (Azzellini 2013, Interview Antillano (2008), Jongerden/Hamdi 2013, Ramírez 2003, Sitrin/ Azzellini 2014: 32-34).

Autonomie bedeutet aber nicht, jede Interaktion mit staatlichen Institutionen zwangsläufig abzulehnen. Je dauerhafter eine Praxis angelegt ist und je mehr Räume sich mit der eigenen Raumproduktion überlagern, desto unausweichlicher ist eine Interaktion. Auch die Platzbesetzungen interagierten mit Behörden und Institutionen. RBA sind noch viel mehr auf Interaktion angewiesen. Sie müssen einen juristischen Prozess (Schließung des Betriebes und Entlassung) in einen politischen Prozess verwandeln. Dafür ist meist an irgendeiner Stelle die Intervention staatlicher Institutionen notwendig. Ein Großteil der RBA in Argentinien, Brasilien, Uruguay und Venezuela und die beiden Fabriken in Frankreich haben eine staatliche Intervention erlebt, von der Enteignung der Ex-Eigentümer[4] bis zu direkten Finanzierungen oder Bürgschaften. Doch bezüglich ihrer Diskussionen, Entscheidungen und Ziele behalten sich alle RBA ihre Autonomie vor (Azzellini 2013; 2014; 2015b, Chedid et al. 2013, PFA 2010: 71, Rieiro 2015).

Im Falle der lokalen Selbstverwaltung beginnt die Autonomie damit, dass von Chiapas über Venezuela bis Kurdistan die Bewohner_innen der Communitys von der kleinsten Einheit bis zur Kommune das Bezugsterritorium und die Zugehörigkeit selbst festlegen (Azzellini 2013, Flach/Ayboğa/ Knapp 2015, Interview Abel (1998), Ramírez 2003). Die Kommunen beziehen sich auf den (relationalen) sozio-kulturell-ökonomischen Raum, der sich aus dem Alltag ableitet und nicht auf den bestehenden politisch-administrativen Raum (Harvey 2006: 119-148). Die beiden Räume überlagern sich und ihr Verhältnis ist meist von Konflikt geprägt. Während in Mexiko und in Kurdistan aufgrund der Konflikt- beziehungsweise Kriegssituation eine Interaktion mit dem Staat (oder mit Staaten im Falle Kurdistans) jenseits des Konflikts kaum existiert, fordern und erhalten Kommunale Räte und comunas in Venezuela 
finanzielle Unterstützung vom Staat. Sie sehen den Zugriff auf den gesellschaftlich produzierten Reichtum als ein Recht an, bestehen aber gegenüber staatlichen Institutionen und Parteien auf ihrer Autonomie. In der Durchsetzung ihrer Vorstellungen und Entscheidungen geraten sie häufig in Konflikt mit staatlichen Institutionen, die nicht effektiv arbeiten beziehungsweise eigene Prioritäten durchsetzen oder die Selbstverwaltungsstrukturen kooptieren wollen (Azzellini 2013; 2014). Auch aus diesem Grund sehen die meisten comunas eine sozio-produktive Entwicklung als notwendig an, um nicht mehr auf den Staat angewiesen zu sein (Azzellini 2013; 2014, Azzellini/Ressler 2010, Interview Figuera León (2015), Interview Jiménez (2012)).

Der Selbstorganisierung wird in der Regel mit Gewalt begegnet, wenn sie die Hoheit des Staates und das Privateigentum allgemein praktisch in Frage stellt. Die Plätze wurden gewaltsam geräumt, wenn sie nicht zuvor verlassen wurden. RBA müssen sich Räumungsversuchen der Polizei und zum Teil auch der Gewalt der Ex-Eigentümer_innen widersetzen. Auf die lokalen Selbstverwaltungen reagiert der Staat - außer in Venezuela - immer mit Repression (Azzellini 2013, 2014, Flach/Ayboğa/Knapp 2015: 283-315, Gasparello 2009, Interview Abel (1998), Jongerden/Hamdi 2013: 170, Ramírez 2003).

Die Erklärung des Basisaktivisten und Soziologen Andrés Antillano aus Caracas zu Venezuela beschreibt auch das gängige Verhältnis der neuen globalen Bewegungen, RBA und Kommunen zum Staat und seinen Institutionen:

„Wir haben immer gesagt, dass wir vorwärts schreiten müssen im Aufbau einer neuen Gesellschaft, mit dem Staat, ohne den Staat, gegen den Staat. Das heißt das Verhältnis mit dem Staat definieren nicht wir, sondern es wird von der Bereitschaft des Staates definiert, sich den Interessen des Pueblo[5] unterzuordnen“(Interview Antillano 2008).

\section{Demokratische Entscheidungsfindung und Raumproduktion}

Die Raumproduktion von Platzbesetzungen, RBA und Kommunen ist durch horizontale und konsensuale Demokratie geprägt. Die Entscheidungsfindung als kollektiven Prozess zu organisieren stärkt Kooperation und Vertrauen. Einfache Mehrheitsentscheidungen hingegen produzieren sich gegenüberstehende Lager. Im Falle eines gemeinsamen Projekts ist aber entscheidend, dass es möglichst breit getragen wird. Die Mechanismen der Entscheidungsfindung sind somit zentral für die Raumproduktion. Die „räumlichen Dynamiken der Besetzung in einer Zelt-Stadt machen das Zusammenleben zur Notwendigkeit. Dies nährt die verschiedenen Stile partizipativer Politiken der Versammlung, welche ein kreatives Mittel bietet, Konfrontation in Kooperation zu verwandeln“ (Arenas 2014: 435). Die Versammlung steht bei Platzbesetzungen wie auch in RBA und Kommunen im Mittelpunkt. Sie ist nicht wie in der liberalen Demokratie der Ort der Ideenkonkurrenz, sondern des kollektiven Denkens und des Aufbaus. Wie Ayelen aus Madrid erläutert:

„Es ist nicht so, dass jeder etwas anderes denkt und wir das alles zusammen werfen. Es geht darum, von Anfang an zusammen etwas aufzubauen, das es vorher nicht gegeben hat [...]. Es geht nicht 
ums Überzeugen, sondern darum, gemeinsam etwas aufzubauen“. (Interview Lozada 2012)

In der gleichen Situation sind auch RBA und Kommunen. Im Betrieb oder in der Nachbarschaft ist die Notwendigkeit des Zusammenlebens existenzieller als auf den Plätzen (Azzellini 2015b, Azzellini/Ressler 2010, Ramírez 2003, Sitrin/Azzellini 2014).

Konsens bedeutet für gewöhnlich nicht Einstimmigkeit, sondern verweist auf einen Prozess, in dem alle Stimmen zu Gehör kommen und auf eine gemeinsame Position hingearbeitet wird. Dafür braucht es zunächst einmal Zeit. Es kommen aber auch zahlreiche Instrumente zum Einsatz, vor allem auf den Plätzen: Moderation, Redelisten mit unterschiedlichen Quotierungen, Redezeitbeschränkungen, Zusammenfassungen, Handzeichen, um Stimmungen auszudrücken und Ablehnung, die keine Beschlüsse blockiert. Auf manchen Plätzen, wie etwa in Madrid, führte ein dogmatisches Beharren einiger Teilnehmer_innen auf völligem Konsens öfters zur Entscheidungsunfähigkeit. Die konsensuale Entscheidungsfindung ist zentral, um den Raum zu erzeugen, der die breite Partizipation ermöglicht, zugleich kann sie aber auch blockierend wirken. Dies war jedoch seltener ein Problem, als die Kritik suggeriert (Harvey 2012: 156, Teivainen 2016). Entscheidungen wurden auf vielen Plätzen mit überwältigenden Mehrheiten getroffen, einige führten auch Sprecherräte ein (Sitrin/Azzellini 2014: 135-140; 173-174).

In RBA wird meist versucht, einen Konsens zu erzielen. Kleinere Betriebe entscheiden in Argentinien nach dem Konsensprinzip (Interview Ruggeri (2015)), ebenso die RBAs in Europa (Azzellini 2015b). In Venezuela kann ich dies für zehn und in Uruguay für zwei kleinere Betriebe, die ich besucht habe, bestätigen. In Brasilien scheint es ähnlich zu sein, allerdings lassen die Daten keine endgültigen Schlüsse zu. In größeren Betrieben wird zwar auch meist konsensual diskutiert, aber häufiger durch große Mehrheiten entschieden (Chedid et al. 2013: 114-132, Interview Ruggeri (2015)).

In der lokalen Selbstverwaltung in Venezuela (Azzellini 2010: 271-300; Azzellini/Ressler 2010, Interview Jiménez (2012)) wie auch in Chiapas (Interview Abel (1998), Ramírez 2003) wird auf Konsens hin diskutiert. „Nicht die Demokratie, die erdrückt, wo es einen Besiegten und einen Sieger gibt“, werde praktiziert, so Rafael Falcón in einem Workshop zur Gründung kollektiver Betriebe einer comuna in Caracas, „sondern eine Konsensdemokratie, wo wir uns alle in den Entscheidungen wiederfinden können“(Azzellini/Ressler 2010). Über einzelne Entscheidungen wird auch abgestimmt, aber selten mit einfacher Mehrheit entschieden. In RBA wie auch in den lokalen Selbstverwaltungen werden Räte, Sprecher_innen, Koordinator_innen, und Verantwortliche gewählt. Sie haben meist keine (oder nur limitierte) Entscheidungsgewalt und können von der Basis wieder abgesetzt werden. Dies hat sich in den Selbstverwaltungsstrukturen in Venezuela (Azzellini 2013), Chiapas (EZLN 2016, Vergara-Camus 2014) und Guerrero (Gasparello 2009) bewährt.

\section{Netzwerke und räumliche Konfigurationen}

Die demokratische Selbstverwaltung erzeugt neue soziale Beziehungen, die von Kooperation, Solidarität und gegenseitiger Unterstützung geprägt sind. 
Die Gemeinsamkeiten zwischen verschiedenen Orten und unterschiedlichen Praktiken haben nicht nur ideellen Charakter. Es wird gegenseitig aufeinander Bezug genommen und es existieren direkte Kontakte zwischen Beteiligten (Roos/Oikonomakis 2014: 128-131, Sitrin/Azzellini 2014). Durch die Verknüpfung anhand von Netzwerken entstehen kulturelle und räumliche Konfigurationen (Escobar 2001, Routledge 2015). Zwischen den Plätzen in Madrid und Kairo bestanden Videoübertragungen; Occupy Gezi lehnte sich schon im Namen an Occupy an; Platzbesetzer_innen aus Spanien waren an Occupy in den USA beteiligt; Athen grüßte Madrid und 15-M und OccupyAktivist_innen besuchten Paris.

Occupy Chicago unterstützte die Arbeiter_innen der RBA „New Era Windows \& Doors“ bei der Besetzung, blockierte eine mögliche Räumung und versorgte die eingeschlossenen Arbeiter_innen mit Lebensmitteln (Lydersen 2017). Für die RBA Kazova in Istanbul war die Unterstützung durch Occupy Gezi und die daraus entstandenen Stadtteilversammlungen entscheidend (Azzellini 2015b). New Era hatte auch Arbeiter_innen aus argentinischen RBA zu Besuch, ebenso eine Mehrheit der RBA in Europa (Azzellini 2015b). Dimitris Koumatsioulis, Arbeiter der RBA Vio.Me. in Thessaloniki, Griechenland, berichtet: „Selbstverständlich hatten wir auch die Genossen aus den argentinischen Fabriken hier, wir haben lange Diskussionen geführt und sie haben uns geholfen, unseren Horizont zu erweitern und unsere Stimmung zu heben. Selbstverständlich haben sie uns auch von allen Schwierigkeiten berichtet [...] und wer uns alles bekämpfen wird“. (Azzellini/Ressler 2015b)

Zwischen italienischen und griechischen RBA und Bewegungen bestehen direkte Verbindungen, Besuche und ein Austausch über Kampfformen (Vogiatzoglou 2015: 24-25). Die Solidarität unter RBA ist generell groß. 82,3 Prozent der argentinischen und 71 Prozent der brasilianischen RBA haben von anderen RBA Unterstützung erhalten (Azzellini 2015a; 2015c, Chedid et al. 2013: 161-166, PFA 2010: 80). In Argentinien, Brasilien, Uruguay und Venezuela existieren landesweite Organisationen und Netzwerke, in denen die RBA zusammenkommen. Darüber hinaus besteht auch eine internationale Vernetzung von RBA.

Die meisten RBA bilden Netzwerke mit anderen Bewegungen sowie mit sozialen und politischen Organisationen. In Argentinien stellt mehr als die Hälfte der RBA permanent Raum für andere Initiativen zur Verfügung (Ruggeri 2010, Sitrin 2012). In Europa sind alle RBA mit anderen Bewegungen verknüpft (Azzellini 2015b, Kokkinidis 2015). RiMaflow in Mailand ist lokal verankert, unterstützt Arbeitskämpfe in der Umgebung und steht darüber hinaus mit sozialen Bewegungen in Italien und international in Kontakt. RiMaflow ist Teil des Comunia-Netzwerkes, das den RBA mit besetzten Bauernhöfen, einem besetzten Park, besetzten sozialen Zentren und solidarökonomischen Akteuren zusammenbringt. Zwischen ihnen bestehen soziale, politische und auch ökonomische Beziehungen. In Venezuela bestehen enge Verbindungen zu Kommunalen Räten und comunas. In einigen Fällen kam es zu gemeinsamen Besetzungen (Azzellini 2016).

Auf internationaler Ebene bestehen ein gegenseitiges Anerkennen, Bezugnahme, Erfahrungsaustausch und gelegentlich Kooperation. Es ist aber keine internationale Koordination, neue Internationale oder Einheitsfront. 
Kooperation und Koordination findet wesentlich auf lokaler, regionaler und nationaler Ebene statt.

Die Kommunen in Chiapas, Venezuela und Kurdistan verstehen sich explizit als antikapitalistisch (Azzellini 2013, Jongerden/Hamdi 2013: 172, Ramírez 2003). In allen werden kollektive und selbstverwaltete Arbeits- und Produktionsformen aufgebaut. Die produktiven Projekte der comunas in Venezuela verfolgen die Intention, die Produktionsverhältnisse zu transformieren und das kapitalistische Wirtschaftsmodell zu überwinden (Interview Jiménez (2012)). Für Chiapas trifft dies ebenfalls zu. Die Koordination des landwirtschaftlichen Anbaus und des Vertriebes der Produktion dient dort auch einem Ausgleich von räumlichen Ungleichheiten (EZLN 2016). Auch in Rojava, der kurdischen Region auf syrischem Staatsgebiet, wird seit 2012 und vor allem seit 2014 verstärkt der Aufbau von Kooperativen in allen Bereichen gefördert (Flach/Ayboğa/Knapp 2015: 251-253). In Venezuela sind die comunas seit 2010 dazu übergegangen, sogenannte Unternehmen Kommunaler Sozialer Produktion (EPSC) zu gründen. Dabei handelt es sich um Kooperativen, die von den Arbeiter_innen und der comuna verwaltet werden (Azzellini 2013, Interview Jiménez (2012)).

\section{Schlussbemerkung}

Alltagspolitik wird an konkreten Orten praktisch. Die Makrobedingungen des Kapitals strukturieren den Raum und reproduzieren sich an jedem Ort. Politische Entscheidungen werden an konkreten Orten zur Realität - und ebenso auch der Widerstand (Harvey 1995). Die Platzbesetzungen sind ein Jetztzeit-Fenster der neuen globalen Bewegungen. In ihnen konzentrieren und potenzieren sich Praktiken, Produktion und Werte, die für die neuen Bewegungen prägend sind (Arenas 2014, Ressler 2012, Roos/ Oikonomakis 2014, Sitrin/Azzellini 2014). RBA und Kommunen können wiederum als Ausübung der Prinzipien der Platzbesetzungen als dauerhaftes Projekt im konkreten Alltag angesehen werden. Das Potenzial dieser Praktiken liegt auch darin, dass sie sich durch die massive Partizipation von Personen auszeichnen, die zuvor nicht an sozialen und politischen Mobilisierungen beteiligt waren. Alle Quellen, ganz gleich ob zu Argentinien, Ägypten, Griechenland, Spanien, der Türkei, Venezuela oder den USA, unterstreichen ganz wesentlich die - den Kontexten entsprechende - soziale, politische, ethnische und so weiter Breite der Beteiligung (Arenas 2014, Azzellini 2015b, Byrne 2012, Graeber 2012, Roos/Oikonomakis 2014, Sitrin 2012, Sitrin/Azzellini 2014, Yörük 2014).

Die jüngste Platzbewegung, die am 31. März 2016 in Paris begann und sich in kürzester Zeit auf über 50 Städte in Frankreich ausdehnte, wurde ausgelöst durch Proteste gegen die Reform der Arbeitsgesetzgebung. Die Besetzungen waren „in sehr ähnlicher Weise wie die der 15-M-Bewegung in Spanien strukturiert, mit partizipativen Entscheidungsstrukturen und in der Überzeugung, dass in einem demokratischen Prozess Menschen viel mehr zu sagen haben sollten. Es ist das organisierte Chaos, mit kostenlosem Essen, professionellen Sound-Systemen, einer Webseite, Live Streaming und einem Medien-Zelt, aber mit niemandem, der die Leitung hat“(Cossar-Gilbert 2016). Die Bewegung „nuit debout“ kam den von langer 
Hand geplanten Gewerkschaftsprotesten zuvor. Die Gewerkschaften wurden gezwungen mitzuziehen. Zugleich aber ging in Frankreich die Ausweitung der Kämpfe und die Zusammenarbeit der neuen Mobilisierungen und traditioneller Akteure (vor allem Gewerkschaften) weiter als in anderen vorangehenden Bewegungen (Syrovatka 2016).

Den Platzbesetzungen, RBA und Kommunen gelingt es, das hegemoniale räumliche Vorstellungsdenken zu überwinden und umzuformulieren. Dafür sind Autonomie und die demokratische Entscheidungsfindung zentral. In den neu geschaffenen Räumen erfolgt eine Freisetzung menschlicher Kreativität. Die Vielfalt der Initiativen und Debatten auf den Plätzen zeugt genauso davon wie die Vielfalt der Aktionen, die von ihnen ausgingen (Sitrin/ Azzellini 2014). Was auf den Plätzen im Kleinen geschieht, ereignet sich in der lokalen Selbstverwaltung im größeren Maßstab. Zahlreiche Initiativen entstehen. Zuvor unmögliche Vorhaben werden angegangen und realisiert, von Bildung und Gesundheitsversorgung über die Gründung von kollektiven Betrieben und Medien bis zu Not- und Verteidigungsmaßnahmen, die es ermöglichen, stärkeren Feinden zu widerstehen (Azzellini 2013, Flach/ Ayboğa/Knapp 2015, Klein 2015, Ramírez 2003, Vergara-Camus 2014). In den Betrieben unter Arbeiterkontrolle ist ebenfalls ein „großes Freilassen menschlicher kreativer Energie" (Wallis 2012: 21) zu beobachten. Es werden Produktionsprozesse verbessert, neue Produkte erfunden, Ersatzteile selbst hergestellt, neue Arbeiten erlernt und kulturelle Aktivitäten integriert (Azzellini 2014; 2015b, Azzellini/Ressler 2014; 2015a; 2015b, CDER 2014, PFA 2010, Sitrin 2012).

Die Bewegungen, um die es hier geht, haben ihre Fähigkeit gezeigt, die Kapitalakkumulation zu stoppen (Harvey 2014: 3), indem sie Räume produzieren, die nicht vom Kapital geformt sind und in denen die Gesetze des Kapitals nicht oder nur bedingt gelten. Der Metabolismus des Kapitals (Mèszaros 2009) wird von Platzbesetzungen, RBA und Kommunen in unterschiedlicher Weise und in unterschiedlichem Ausmaß gestört und auBer Kraft gesetzt. Dies bedeutet nicht, dass dadurch alle kapitalistischen Verhältnisse überwunden würden. Kapitalismus ist ein soziales Verhältnis und damit nicht durch einzelne ,Maßnahmen“ zu beseitigen. Das Kapital ist auf die Strukturierung des Raumes zur Sicherstellung der Umschlagszeiten für die Reproduktion des Kapitals und seine Akkumulation angewiesen. Dies wird von den Bewegungen in den entsprechenden Kontexten verlangsamt beziehungsweise außer Kraft gesetzt.

Auf den Plätzen bestanden soziale Beziehungen, welche die Kapitalakkumulation außer Kraft gesetzt haben. Die sozialen Beziehungen auf den Plätzen basierten nicht auf den Regeln der Kapitalakkumulation. Das bedeutet nicht, dass sie die Kapitalakkumulation in der Gesellschaft oder global außer Kraft gesetzt oder gar den Kapitalismus überwunden hätten. Die RBA funktionieren im Kapitalismus, ohne sich nach allen seinen Regeln zu richten. Sie produzieren für gewöhnlich, ohne Kapitalakkumulation zu betreiben und sich auf die Erhöhung des Mehrwerts zu konzentrieren - was nicht verhindert, dass sie mit dem kapitalistischen Markt in Interaktion treten und dort bestehen müssen.

„Wir nehmen jeden Monat einen Teil unserer Arbeitszeit und investieren sie in den Kampf, zur Unterstützung anderer Kämpfe. Gemäß der kapitalistischen Logik ist das verlorene Zeit“, so Ernesto des RBA Druckerei 
Chilavert, Buenos Aires (Interview Ernesto (2015)). Eine Haltung, die nicht unüblich ist in RBA. Die RBA zeigen die konkrete Möglichkeit auf, eine andere Form der Ökonomie aufzubauen. Trotz widriger Bedingungen haben sie eine längere Lebensdauer als herkömmliche Betriebe. Von 205 RBA, die 2010 in Argentinien untersucht wurden, schlossen bis Ende 2013 nur sechs ihre Pforten (CDER 2014: 10, 13). Dabei bleiben die RBA - in Argentinien, Brasilien und Uruguay - ihren Prinzipien der Gleichheit und der demokratischen Selbstverwaltung treu (Chedid et al. 2013, Ozarow/Croucher 2014: 990, Rieiro 2015). In Chiapas, Guerrero, Kurdistan und Venezuela ist zu sehen, wie unterschiedliche Erfahrungen der Selbstverwaltung zu einem alternativen antikapitalistischen Gesellschaftsmodell zusammengeführt werden.

Die Gemeinsamkeiten, Verbindungen und Bezüge zwischen neuen globalen Bewegungen, RBA und Kommunen lassen den Schluss zu, sie einer geteilten nicht-staatszentrierten Transformationsstrategie zuzuordnen. In ihren kulturellen und räumlichen Konfigurationen stellen die Bewegungen diese Zusammenhänge auch selbst her. Ebenso lässt sich feststellen, dass Platzbesetzungen, RBA und Kommunen keine vorrübergehenden Erscheinungen sind - letztere haben sich in den vergangenen Jahren weiterverbreitet und konsolidiert, während sich neue massive Platzbesetzungen an neuen Orten ereignet haben.

Wie Spanakos (2016) aus der Perspektive der komparativen Politikwissenschaften bezüglich der partizipativen Praktiken der Linksregierungen in Lateinamerika und der Platzbesetzungen bemerkt: Auch wenn eine Institutionalisierung nicht sofort erkennbar sei, könne nicht behauptet werden, es sei nicht zumindest damit begonnen worden, eine neue Ordnung zu errichten, deren institutionelle Form noch nicht festgelegt wurde und auch nicht auf traditionellem Wege festgelegt werden wird.

\section{Endnoten}

[1] Es geht hier nicht um den Akt der Platzbesetzung an sich, eine Praxis die es auch in der Vergangenheit gegeben hat, sondern um die Platzbesetzungen als Ausdruck der neuen globalen Bewegungen. Das sind nicht alle Bewegungen, die in den vergangenen Jahren ähnliche Praktiken angewandt haben. So gehört z. B. das Beispiel des Maidan in der Ukraine, eine Platzbesetzung, die in eine nationalchauvinistische bis faschistische Mobilisierung abkippte, nicht in diese Reihe. Die Anerkennung einer absoluten Gleichheit bei aller Unterschiedlichkeit ist eine der wesentlichen Grundlagen der neuen globalen Bewegungen. Rassistische, faschistische oder nationalchauvinistische Positionen waren auf allen anderen Plätzen ausgeschlossen. Auf dem Maidan waren sie von Beginn an (minoritär) präsent und wurden toleriert. Insofern war der Maidan nicht Ausdruck der neuen globalen Bewegungen.

[2] Ich habe seit 1997 wiederholt mehrere Monate in Chiapas in Autonomen Gemeinden geforscht. Auch in Guerrero und Oaxaca habe ich lokale Selbstverwaltungsstrukturen besucht und interviewt. In Venezuela habe ich von 2003 bis 2012 insgesamt über vier Jahre verbracht und mit Kommunalen Räten, Comunas, dem Nationalen Netzwerk der Kommunard_innen und Arbeiter_innen für Arbeiterkontrolle gearbeitet und dazu geforscht. Seit 2003 habe ich etwa 40 RBA in Venezuela, Argentinien, Uruguay, Italien, Frankreich und Griechenland besucht, Interviews durchgeführt und mich an nationalen und internationalen Konferenzen von RBA-Arbeiter_innen und Forscher_innen beteiligt. Ich habe diverse Occupies in den US besucht (vor allem OWS ab der dritten Woche) und mich 2013 mehrere Monate an der Stadtteilversammlung Occupy Kensington in Brooklyn, New York, beteiligt. Darüber hinaus habe ich Occupy London besucht und war 
seit 2011 mehrmals an verschiedenen Orten in den USA, Spanien und Griechenland, wo ich ebenfalls Interviews geführt habe.

[3] Vgl. Ministerio del Poder Popular para las Comunas y los Movimientos Sociales: http:// consulta.mpcomunas.gob.ve/.

[4] Der Autor verzichtet a. d. S. ausdrücklich auf die gegenderte Form mit dem Hinweis, dass diese aufgrund des geringen Anteils an Frauen unter den Eigentümern bzw. des Fehlens queerer Eigentümer die Geschlechterverhältnisse verschleiere. (Anm. d. Lektorats)

[5] Volk. Hier allerdings im Sinne von „popular“ wie bei Gramsci mit einer klaren Klassendimension.

\section{Autor_innen}

Dario Azzellini ist Soziologe und Politikwissenschaftler. Er arbeitet zu Prozessen sozialer Transformation, popularen Bewegungen, lokaler und Arbeiterselbstverwaltung.

dario@azzellini.net

\section{Literatur}

Albert, Michael (2006): Parecon. Leben nach dem Kapitalismus. Frankfurt am Main: Trotzdem Verlag.

Alexander, Anne / Bassiouny, Mostafa (2014): Bread, Freedom, Social Justice. Workers and the Egyptian Revolution. London: Zed Books.

Ali, Khalid (2012): Precursors of the Egyptian Revolution. In: IDS Bulletin. The Pulse of Egypt's Revolt 43/1, 16-25.

Arenas, Iván (2014): Assembling the multitude: Material geographies of social movements from Oaxaca to Occupy. Environment and Planning D: Society and Space 32, 433-449.

Azzellini, Dario (2010): Partizipation, Arbeiterkontrolle und die Commune: Bewegungen und soziale Transformation am Beispiel Venezuela. Hamburg: VSA.

Azzellini, Dario (2013): The Communal System as Venezuela's transition to Socialism. In: Shannon K. Brincat (Hg.), Communism in the 21st Century, Vol. II: Whither Communism? The Challenges Facing Communist States, Parties and Ideals. Westport: Praeger Publishers.

Azzellini, Dario (2014): Venezuela's social transformation and growing class struggle. In: Susan Spronk / Jeffery R. Webber (Hg.): Crisis and Contradiction: Marxist Perspectives on Latin America in the Global Economy. Leiden: Brill Press, 138-162.

Azzellini, Dario (Hg.) (2015a): An Alternative Labour History: Worker Control and Workplace Democracy. London und New York: Zed Books.

Azzellini, Dario (2015b): Contemporary crisis and workers' control. In: Dario Azzellini (Hg.), An Alternative Labour History. Worker Control and Workplace Democracy. London und New York: Zed Books, 67-99.

Azzellini, Dario (2015c): Besetzen, Widerstand leisten, produzieren. Betriebsbesetzungen in Argentinien, Brasilien, Uruguay und Venezuela. In: Journal für Entwicklungspolitik 31/2, 79-105.

Azzellini, Dario (2016): Workers control and class struggle in state owned companies in Venezuela.In: Latin American Perspectives, onlinefirst, DOI: 10.1177/0094582X16666016.

Azzellini, Dario / Ness, Immanuel (Hg.) (2012): Die endlich entdeckte politische Form. Fabrikräte und Selbstverwaltung von der russischen Revolution bis heute. Köln: Neuer ISP Verlag.

Azzellini, Dario / Ressler, Oliver (2010): Comuna en construcción. Caracas, Berlin und Wien: Film im Eigenverlag, $94 \mathrm{~min}$.

Azzellini, Dario / Ressler, Oliver (2014): Occupy, Resist, Produce - RiMaflow. Berlin und Wien: Film im Eigenverlag, 34 min.

Azzellini, Dario / Ressler, Oliver (2015a): Occupy, Resist, Produce - Officine Zero. Berlin und Wien: Film im Eigenverlag, 33 min.

Azzellini, Dario / Ressler, Oliver (2015b): Occupy, Resist, Produce - Vio.Me.. Berlin und Wien: Film im Eigenverlag, $31 \mathrm{~min}$. 
Beas Torres, Carlos (2007): La batalla por Oaxaca. In: Carlos Beas Torres (Hg): La batalla por Oaxaca. Oaxaca: Yope Power, 21-79.

Benjamin, Walter (1965): Geschichtsphilosophische Thesen. In: Zur Kritik der Gewalt und andere Aufsätze. Frankfurt am Main: Suhrkamp, 78-94.

Biehl, Janet / Bookchin, Murray (1997): The Politics of Social Ecology: Libertarian Municipalism. Montreal: Black Rose Books.

Bloch, Ernst. (1973): Das Prinzip Hoffnung, 3 Bde.. Frankfurt am Main: Suhrkamp.

Bonnet, Alberto (2014): The idea of councils runs through Latin America. In: South Atlantic Quarterly 113/2, 271-283.

Bookchin, Murray (1992): Urbanization. Without. Cities. The Rise and Decline of Citizenship. Montreal: Black Rose Books.

Bookchin, Murray (2015): The Next Revolution: Popular assemblies and the promise of direct democracy. London und New York: Verso.

Byrne, Janet (2012): The Occupy Handbook. New York: Back Bay Books.

Castañeda, Ernesto (2012): The Indignados of Spain: A precedent to Occupy Wall Street. In: Social Movement Studies 11/3, 1-11.

Castells, Manuel (2012): Networks of Outrage and Hope. Cambridge: Polity Press.

CDER (Centro de Documentación de Empresas Recuperadas) (2014): Nuevas Empresas Recuperadas 2010-2013. Buenos Aires: Continente.

Chedid Henriques, Flávio / Moreira Sígolo, Vanessa / Rufino, Sandra / Santos Araújo, Fernanda / Nepomuceno, Vicente / Baptista Girotto, Mariana / Paulucci, Maria Alejandra / Nogueira Rodrigues, Thiago / Rocha Cavalcanti, Maíra / Sardá de Faria, Maurício (2013): Empresas Recuperadas por Trabalhadores no Brasil: Resultados de um Levantamento Nacional. Rio de Janeiro: Editorial Multifoco.

Cossar-Gilbert, Sam (2016): \#NuitDebout: A movement is growing in France's squares. In: ROAR Magazine, 6. April. https://roarmag.org/essays/nuit-debout-republique-occupation/ (letzter Zugriff am 7.4.2016).

Dean, Jodi (2013): Occupy Wall Street: After the Anarchist Moment. In: Leo Panitch / Greg Albo / Vivek Chibber (Hg.), Socialist Register 2013: The Question of Strategy. London: Merlin Press, 52-62.

Demirovic, Alex (2009): Rätedemokratie und das Ende der Politik. In: PROKLA 39/2. http://www.linksnet.de/de/artikel/24699 (letzter Zugriff am 13.3.2016).

Demirovic, Alex (2014): Transformation und Ereignis. Zur Dynamik demokratischer Veränderungsprozesse der kapitalistischen Gesellschaftsformation. In: Michael Brie (Hg.), Futuring. Münster: Westfälisches Dampfboot, 419-435.

Escobar, Arturo (2001): Culture sits in places: Reflections on globalism and subaltern strategies of localization. In: Political Geography 20, 139-174.

Esteva, Gustavo (2009): Otra mirada, otra democracia. http://www.rebelion.org/noticia. php?id=80143 (letzter Zugriff am 13.3.2016).

Esteva, Gustavo (2010): The Oaxaca Commune and Mexico's Coming Insurrection. In: Antipode 42/4, 978-993.

EZLN (Zapatistische Armee der Nationalen Befreiung) (2016): Und in den zapatistischen Gemeinden? http://www.chiapas.eu/news.php?id=8685 (letzter Zugriff am 13.3.2016).

Feigenbaum, Anna / Frenzel, Fabian / McCurdy, Patrick (Hg.) (2013): Protest Camps. London und New York: Zed Books.

Flach, Anja / Ayboğa, Ercan / Knapp, Michael (2015): Revolution in Rojava. Frauenbewegung und Kommunalismus zwischen Krieg und Embargo. Hamburg: VSA.

Gasparello, Giovanna (2009): Policía comunitaria de guerrero, investigación y autonomía. In: Política y Cultura 32, 61-78.

Graeber, David (2012): Inside Occupy. Frankfurt am Main.: Campus.

Hardt, Michael / Negri, Antonio (2009): Commonwealth. Cambridge: Harvard University Press.

Hardt, Michael / Negri, Antonio (2013): Demokratie! Wofür wir kämpfen. Frankfurt am Main: Campus Verlag.

Harvey David (1995): Militant particularism and global ambition: the conceptual politics of place, space, and environment in the work of Raymond Williams. In: Social Text 42, 69-98.

Harvey David (2000): Spaces of Hope. Berkeley und Los Angeles: University of California Press. 
Harvey, David (2006): Spaces of Global Capitalism. Towards a Theory of Uneven Geographical Development. London und New York: Verso.

Harvey David (2012): Rebel Cities: From the Right to the City to the Urban Revolution. London und New York: Verso.

Harvey, David (2014): Foreword. In: Marina Sitrin / Dario Azzellini, They Can't Represent Us!: Reinventing Democracy from Greece to Occupy, London und New York, 1-4.

Holloway, John (2010): Crack Capitalism. London: Pluto Press.

Jongerden, Joost / Hamdi Akkaya, Ahmet (2013): Democratic confederalism as a Kurdish Spring: The PKK and the quest for radical democracy. In: Mohammad M. A., Ahmed / Michael M. Gunter (Hg.), The Kurdish Spring: Geopolitical Changes and the Kurds. Costa Mesa: Mazda Publishers, 163-185.

Juris, Jeffrey S. / Ronayne, Michelle / Shokooh-Valle, Firuzeh / Wengronowitz, Robert (2012): Negotiating Power and Difference within the 99\%. In: Social Movement Studies $11 / 3,1-7$.

Klein, Hilary (2015): Compañeras: Zapatista Women's Stories. New York: Seven Stories Press.

Kokkinidis, George (2015): Spaces of possibilities: Workers' self-management in Greece. In: Organization 22/6, 847-871.

Lopes de Souza, Marcelo (2014): „Towards a libertarian turn? Notes on the past and future of radical urban research and praxis“. In: City 18/2, 104-118.

Linebaugh, Peter (2014): Stop, Thief! The Commons, Enclosures, and Resistance. Oakland: PM Press.

Lydersen, Kari (2017): Revolts on Goose Island: A long fight pays off for Chicago window factory workers. In: Dario Azzellini / Michael G. Kraft (Hg.), The Class Strikes Back. SelfOrganized Workers' Struggles in the 21st Century. Amsterdam: Brill, i. E.

Maeckelbergh, Marianne (2011) Doing is believing: Prefiguration as strategic practice in the Alter Globalization Movement. In: Social Movement Studies 10/1, 1-20.

Mason, Paul (2013): Why is it still kicking off everywhere? The new global revolutions. London / New York: Verso.

Massey, Doreen (2009): Concepts of space and power in theory and in political practice. In: Documents d'analisi geografica 55, 15-26.

McAdam, Doug / Tarrow, Sidney / Tilly, Charles (2001): Dynamics of Contention. Cambridge: Cambridge University Press.

Mészáros, Istvan (1995): Beyond Capital. Towards a Theory of Transition. London: The Merlin Press.

Muñoz Ramírez, Gloria (2003): 20 + 10 Das Feuer und das Wort. Münster: Unrast Verlag.

Novaes, Henrique T. / Sardá de Faria, Maurício (2014): ¿Para donde van las empresas recuperadas por los trabajadores brasileños? In: Andrés Ruggeri / Henrique Novaes / Mauricio Sardá de Faría (2014): Crisis y Autogestión en el Siglo XXI. Cooperativas y Empresas Recuperadas en Tiempos de Neoliberalismo. Buenos Aires: Continente, 75-90.

Ozarow, Daniel / Croucher, Richard (2014): Workers' self-management, recovered companies and the sociology of work. In: Sociology 48/5, 989-1006.

PFA (Programa Facultad Abierta) (2010): Las Empresas Recuperadas en la Argentina: Informe del Tercer Relevamiento. Buenos Aires: University of Buenos Aires.

Polletta, Francesca (2002): Freedom is an Endless Meeting. Chicago: University of Chicago Press.

Porcaro, Mimmo (2013): Occupy Lenin. In: LuXemburg 1/2013, 132-139.

Ressler, Oliver (2012): Take the Squares. Wien: Film im Eigenverlag, $88 \mathrm{~min}$.

Rieiro, Anabel (2015): Collective self-management and cocial Classes: The case of enterprises recovered by their workers in Uruguay. In: Dario Azzellini (Hg.), An Alternative Labour History: Worker Control and Workplace Democracy. London: Zed Books, 273-297.

Roberts, Alasdair (2012): Why the Occupy Movement Failed. In: Public Administration Review 72/5, 754-762.

Roos, Jérôme E. / Oikonomakis, Leonidas (2014): They don't represent us! The global resonance of the Real Democracy Movement from the Indignados to Occupy. In: Donatella della Porta / Alice Mattoni (Hg.): Spreading Protest: Social Movements in Times of Crisis. Colchester: ECPR Press, 117-136.

Ross, Kristin (2015): Comunal Luxury. The Political Immaginary of the Paris Commune. New York und London: Verso. 
Roth, Karl Heinz (2008): Globale Krise - Globale Proletarisierung - Gegenperspektiven. Zusammenfassung der ersten Ergebnisse. http://www.wildcat-www.de/aktuell/ao68_ khroth_krise.htm (letzter Zugriff am 13.3.2016).

Routledge, Paul (2015): Geography and social movements. In: Donatella della Porta / Mario Diani (Hg.): The Oxford Handbook of Social Movements. New York: Oxford University Press, 383-395.

Ruggeri, Andrés (2010): Autogestión obrera y empresas recuperadas, límites y potenciales en el capitalismo neoliberal globalizado. Buenos Aires: Universidad de Buenos Aires.

Ruggeri, Andrés (2014): Crisis y Autogestión en el Siglo XXI. In: Andrés Ruggeri / Henrique Novaes / Mauricio Sardá de Faría, (2014), Crisis y Autogestión en el Siglo XXI. Cooperativas y empresas recuperadas en tiempos de neoliberalismo. Buenos Aires: Continente, 13-26.

Sardá De Faria, Maurício / Novaes, Henrique T. (2012): Die Zwänge der Arbeiterkontrolle bei besetzten und selbstverwalteten brasilianischen Fabriken. In: Azzellini, Dario / Ness, Immanuel (Hg.): Die endlich entdeckte politische Form. Fabrikräte und Selbstverwaltung von der russischen Revolution bis heute. Köln: Neuer ISP Verlag, 503-527.

Schumacher, Juliane / Osman, Gaby (2012): Tahrir und kein Zurück. Ägypten, die Bewegung und der Kampf um die Revolution. Münster: Unrast.

Sitrin, Marina (2006): Horizontalism. Voices of Popular Power in Argentina. Oakland: AK Press.

Sitrin, Marina (2012): Everyday Revolutions. London und New York: Zed Books.

Sitrin, Marina (2013): Occupy Trust: The role of emotion in the new movements. In: Cultural Anthropology Online. http://culanth.org/fieldsights/76-occupy-trust-the-roleof-emotion-in-the-new-movements (letzter Zugriff am 13.3.2016).

Sitrin, Marina / Azzellini, Dario (2014): They Can`t Represent Us! Reinventing Democracy from Greece to Occupy. London und New York: Verso.

Sowers, Jeannie / Toensing, Chris (2012): The Journey to Tahrir: Revolution, Protest and Social Change in Egypt 1999-2011. London und New York: Verso Books.

Spanakos, Anthony P. (2016): Institutionalities and political change in Bolivarian Venezuela. In: Anthony P. Spanakos / Francisco Panizza (Hg.): Conceptualizing Comparative Politics. New York: Routledge, 215-242.

Syrovatka, Felix (2016): Nuit Debout: Frankreich gerät in Bewegung! In: PROKLA 46/2, 317-323.

Teivainen, Teivo (2016): Occupy representation and democratise prefiguration: Speaking for others in global justice movements. In: Capital \& Class 40/1, 19-36.

Van de Sande, Mathijs (2013): The Prefigurative Politics of Tahrir Square: An Alternative Perspective on the 2011 Revolutions. In: Res Publica 19/3, 233-239.

Vergara-Camus, Leandro (2014): Land and Freedom. London: Zed Books.

Vogiatzoglou, Markos (2015): Workers' transnational networks in times of austerity. Italy and Greece. In: Transfer: European Review of Labour and Research 21/2, 215-228.

Wainwright, Hilary (2012): Griechenland: Syriza weckt Hoffnungen. In: LuXemburg 3/2012, 118-25.

Wallis, Victor (2012): Arbeiterkontrolle und Revolution. In: Azzellini, Dario / Ness, Immanuel (Hg,): Die endlich entdeckte politische Form. Fabrikräte und Selbstverwaltung von der russischen Revolution bis heute. Köln: Neuer ISP Verlag, 20-45.

Wolff, Richard (2012): Democracy at Work: A Cure for Capitalism. Chicago: Haymarket Books.

Yörük, Erdem (2014): The long summer of Turkey: The Gezi Uprising and its historical roots. In: South Atlantic Quarterly 113/2, 419-426.

Zelik, Raul / Tauss, Aaron (Hg.) (2013): Andere mögliche Welten? Krise, Linksregierungen, populare Bewegungen: Eine lateinamerikanisch-europäische Debatte, Hamburg: VSA.

\section{Interviews}

Interview Abel (1998): Interview mit Abel, Verantwortlicher für Bereich Produktion der Autonomen Gemeinde „17 de Noviembre“, Autonomer Landkreis „Torbellino de nuestras palabras“ (ex-Morelia), durchgeführt im Februar 1998 von Dario Azzellini in Chiapas, Mexiko. 
Interview Antillano (2008): Interview mit Andrés Antillano, Soziologe und Aktivist der Urbanen Landkomittees (CTU), durchgeführt am 20.4.2008 von Dario Azzellini in Caracas, Venezuela.

Interview Ernesto (2015): Interview mit Ernesto, Arbeiter, RBA Kooperative Chilavert (Druckerei), durchgeführt am 13.2.2015 von Dario Azzellini in Beunos Aires, Argentinien.

Interview Figuera León (2015):, Interview mit Adys Figuera León, Promotorin für populare Macht, Kommunaler Rat Las Charras, Kommune Los 7 Pilares Socialistas, durchgeführt am 11.2.2012 von Dario Azzellini in Anaco, Anzoátegui, Venezuela.

Interview Jiménez (2012): Interview mit Atenea Jiménez, 38 Jahre, Nationales Netzwerk der KommunardInnen Venezuelas, durchgeführt am 14.2.2012 von Dario Azzellini in Belomonte, Caracas, Venezuela.

Interview Lozada (2012) Interview mit Ayelen Lozada,15-M Madrid, Physiotherapeutin, durchgeführt am 8.1.2012 von Dario Azzellini in Madrid, Spanien.

Interview Ruggeri (2015): Interview mit Andrés Ruggeri, Universidad de Buenos Aires, Direktor des CDER, durchgeführt am 15.1.2015 von Dario Azzellini in Buenos Aires, Argentinien.

\section{"This is a process not a protest". Global urban protests, workplace occupation for production under workers' control and local self-government.}

The article connects the square occupations since 2011 with the workplaces under workers' control, which started to appear in Latin America around 2000 and then spread out from there, and with forms of local self-management. Beyond all differences these practices all build spaces for the production of social alternatives. I point out the shared characteristics and argue that they are part of global non-state-centered perspectives of social transformation. These practices of the new global movements display a growing tendency towards direct democracy, self-organization and autonomy. Contrary to the critique regarding a supposed lack of strategy I show how the strategy consists precisely in experimenting and developing the aspired societal changes in the self-produced spaces. The participants of these practices do not see them as a niche but as an alternative model for the whole of society. The practices are connected among each other through networks and form new spatial configurations. 